\title{
THEORY AND DESIGN OF A PROPELLER TURBINE
}

(Read before the General Meeting of the Engineering Society, S. M. E. Section, Nov. 4, 1927)

By

Otogoro Mryagr, Kôgakuhakusi, Member.

\begin{abstract}
The clearance space is a wide open space lying between the guide vanes and the runner, and the flow in it being a potential one, the form of the casing and that of the hub of the runner as well as the stream lines in it should obey ordinary hydrodynamic laws of irrotationality.

Taking this into consideration, the forms of the casing and the hub are first given, and the stream-line forms in the clearance space are determined by making use of cylindrical co-ordinates. The inflow velocities into the runner are then calculated from the stream lines, and the entrance and exit forms of the runner vanes, to satisfy the condition of minimum hydraulic losses, are determined.

The expressions for the developing moment and the power are deduced, and finally a graphical solution, in order to simplify the above calculations relating to the vanes, is introduced.
\end{abstract}

\section{Flow in the Clearance AND the Form OF RUNNER VANE}

IN order to study the theory of a propeller turbine, the first thing to know is the motion of the water and its path in the clearance space lying between the guide vanes and the runner, which is a wide open space in which the flow changes its direction from radially inwards to axially downwards, or to some other direction, as shown in Fig. 1.

The flow in the clearance space being a natural or a potential one, the irrotational conditions must be fulfilled in it, which are written, in cylindrical co-ordinates with axes $r$ and $z$, as follows :

$$
\frac{\partial w}{\partial \theta}-\frac{\partial(v r)}{\partial z}=0, \quad \frac{\partial s}{\partial z}-\frac{\partial w}{\partial r}=0, \quad \frac{\partial(v r)}{\partial r}-\frac{\partial s}{\partial \theta}=0
$$

Here $s, v$ and $w$ indicate the radial, circumferential and axial velocities respectively and $\theta$ shows the anguiar position about the axis of $z$ which is taken downwards as positive.

The flow being symmetrical about the axis of $z$, the terms containing $\theta$ in the above equations should vanish, and the resulting equations become

$$
\frac{\partial(v r)}{\partial z}=0, \quad \frac{\partial s}{\partial z}-\frac{\partial w}{\partial r}=0, \quad \frac{\partial(v r)}{\partial r} 0
$$

[Vol. XXXI, No. 136 
The first and the last of these equations show that the product of vr

should be constant irrespective of $r$ and $z$, and if we denote this

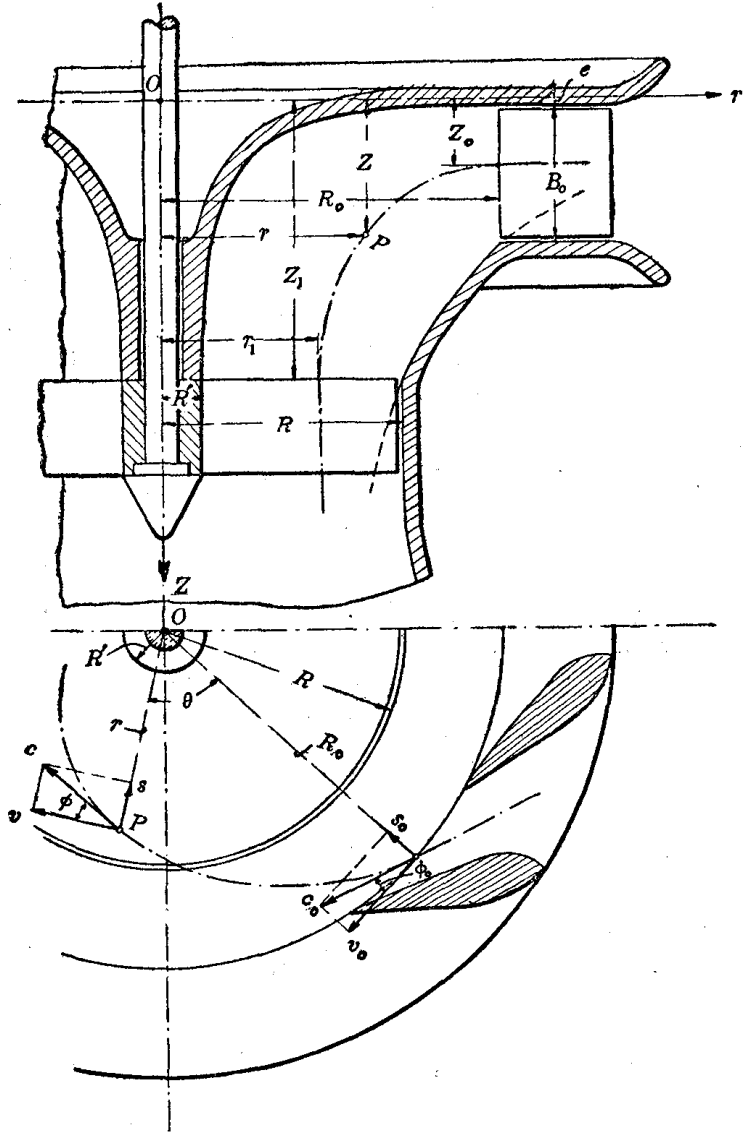

Fig. 1 product by a constant $k_{1}$, we have.

$$
v r=k_{1}
$$

The equation of continuity expressed in the cylindrical coordinates is

$\frac{\partial(s r)}{\partial r}+\frac{\partial(v r)}{r \partial \theta}+\frac{\partial(w r)}{\partial z}=0$

or from the symmetry of flow about the axis of $z$,

$$
\frac{\partial(s r)}{\partial r}+\frac{\partial(w r)}{\partial z}=0, \ldots(3)
$$

The second equation of equations ( 1 ) is

$$
\frac{\partial s}{\partial z}-\frac{\partial w}{\partial r}=0
$$

All kinds of flow satisfying equations (2), (3) and (4) simultaneously are those which are required in the clearace space,

as the flow in it should be continuous and irrotational.

The solution satisfying equations (3) and (4) simultaneously is

$$
s=-a r, \quad w=2 a z,
$$

$a$ being a constant. The corresponding stream line traced on a meridian plane is such a one that the product of $r^{2} z$ is constant, as can easily be obtained $b_{y}$ solving the stream function. If we denote this constant by $k_{2}$, we have

$$
r^{2} z=k_{2}^{(1)}
$$

It follows, therefore, that if the form of the walls of the clearance space be made to satisfy equation (6), which is a kind of a hyperbola

(I) See, for exaniple, H. Loren7, Technische Hydromechanik, p. 357.

Aug. 1928] 
with axes of $r$ and $z$ as its assymptotes, the flow in it is continuous and irrotational.

Assuming the channel walls of the clearance space to be formed hyperbolic to satisfy equation (6), the flow projected on a transverse plane perpendicular to the axis of $z$ will be determined as follows :-

Referring to Fig. 1, let $\phi$ be the angle which the horizontal velocity of the water $c$ makes with the circumferential direction at any point $P$ on the path of the flow, then we have

$$
\tan \varphi=\frac{s}{v}=-\frac{a r^{2}}{k_{1}}
$$

with equations (2) and (5), or since $\frac{s}{v}=\frac{d r}{r d \theta}$, we have

$$
\frac{d r}{r^{3}}=-\frac{a}{k_{1}} d \theta
$$

and its integration is

$$
\frac{1}{2 r^{2}}=\frac{a}{k_{1}} \theta+C
$$

If we trace the path from the exit point of the guide vane, which is at the radius $R_{0}$, then at $r=R_{0}$, we have $\theta=0$, so that we have the constant $C=\frac{1}{2 R_{0}^{2}}$. The above equation then becomes

$$
r=\frac{1}{\sqrt{\frac{2 a}{k_{1}} \theta+\frac{1}{R_{0}^{2}}}}
$$

This is the equation of the path projected on the transverse plane and it gives a kind of a spiral.

Combining equations (6) and (7) we obtain

$$
z=k_{2}\left(\frac{2 a}{k_{1}} \theta+\frac{1}{R_{0}^{2}}\right)
$$

which gives the depth $z$ corresponding to the angle $\theta$. The path in the space can be traced by making use of equations (7) and (8).

Let the notations with the suffix 1 refer to those at the entrance to the runner, which is at the depth $z_{1}$ from the axis of $r$, then we have by equation (5), $w_{1}=2 a z_{1}$.

Assuming most propeiler turbine runners, $z_{1}$ being constant at its [Vol. XXXI, No. 136 
entrance, the axial velocity $w_{1}$ is uniform, so that if $Q$ be the discharge and $R$ and $R^{\prime}$ be the radii of the runner and the hub respectively, we have

$$
w_{1}=\frac{Q}{\pi\left(R^{2}-R^{\prime 2}\right) \rho_{1}}
$$

$\rho_{1}$ being a factor less than unity, giving the ratio of the free total area between the vanes to the vaneless total area at the entrance to the runner.

From the above two equations we get

$$
a=\frac{Q}{2 \pi\left(R^{2}-R^{\prime 2}\right) z_{1} \rho_{1}} .
$$

This determines the value of the constant $\alpha$ used in all the above equations.

If the notations with the suffix 0 refer to those at the exit point of the guide vane, equations (2) and (6) give

and

$$
k_{1}=v_{1} r_{1}=v_{0} R_{0}
$$

$$
k_{2}=r_{1}^{2} z_{1}=R_{0}^{2} z_{0}
$$

where $z_{0}$ is the depth of a stream at the exit point of the guide vane, which passes the runner at the radius $r_{1}$. These determine the values of the constants $k_{1}$ and $k_{2}$ for any specified stream line.

From equations (11) and (12) we obtain

$$
v_{1}=\frac{v_{11} R_{0}}{r_{1}}, \ldots \ldots \ldots \ldots \ldots \ldots \ldots \ldots \ldots \ldots \ldots \ldots \ldots \ldots
$$

and

$$
r_{1}=R_{0} \sqrt{\frac{z_{0}}{z_{1}}} \text {. }
$$

The radial velocity $s$ depends solely on $r$ as shown in equation (5), so that at the exit of the guide vane the radial velocity $s_{0}$ is uniform, and we have

$$
Q=2 \pi R_{0} B_{0} s_{0} \rho_{0},
$$

where $B_{0}$ is the axial width of the guide vane, and $\rho_{0}$ is a factor less than unity, giving the ratio of the free total area between the vanes to the vaneless total area at the exit from the guide vane.

This must be equal to $\pi\left(R^{2}-R^{\prime 2}\right) w_{1} \rho_{1}$, and we have

$$
R_{0} B_{0}=\frac{w_{1} \rho_{1}}{2 s_{0} \rho_{0}}\left(R^{2}-R^{\prime 2}\right)
$$

Aug. 1928] 
or since

$$
\frac{w_{1}}{s_{0}}=\frac{2 a z_{1}}{a R_{0}}=\frac{2 z_{1}}{R_{0}}
$$

$$
B_{0}=z_{1} \frac{\rho_{1}}{\rho_{0}} \frac{R^{2}-R^{\prime 2}}{R_{0}^{2}} \ldots \ldots \ldots \ldots \ldots \ldots \ldots \ldots \ldots
$$

Let $\alpha_{1}$ be the angle which the inflow stream to the runner makes with the transverse plane and which is projected on the tangential plane at the radius $r_{1}$, then we have

$$
\tan \alpha_{1}=\frac{w_{1}}{v_{1}}=\frac{w_{1} r_{1}}{v_{0} R_{0}} .
$$

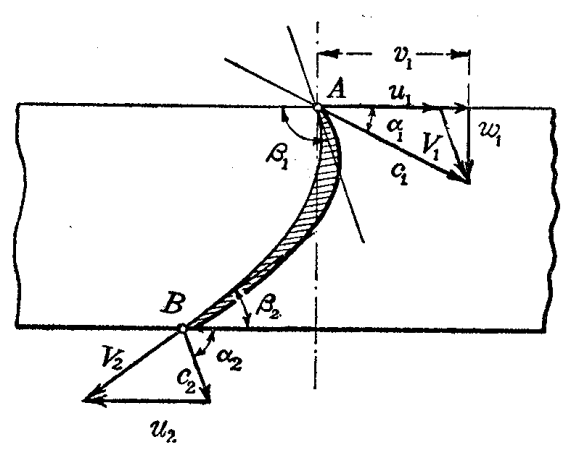

Fig. 2

This determines the absolute inflow direction of the stream projected on the tangential plane at the entrance point of the runner.

Fig. 2 shows the section of the vane cut by the cylindrical surface of the radius $r_{1}$, with the velocity diagram at the entrance point $A$ and the exit point $B$.

Let $c, u$ and $V$ be the absolute velocity of the water, the linear velocity of the rotation of the runner and the velocity of the water relative to the runner respectively at the radius $r_{1}$, with the suffix 1 referring to the entrance and 2 to the exit. Then we have

$$
c_{1}=\sqrt{v_{1}^{2}+w_{1}^{2}}=\sqrt{\left(\frac{v_{0} R_{0}}{r_{1}}\right)^{2}+w_{1}^{2}}, \ldots \ldots \ldots \ldots \ldots \ldots \ldots
$$

and if $\omega$ be the angular velocity of rotation of the runner, since $u_{1}=\omega r_{1}$, we have

$$
V_{1}=\sqrt{\omega^{2} r_{1}^{2}+c_{1}^{2}-2 \omega r_{1} c_{1} \cos \alpha_{1}}
$$

In order that the stream can enter into the runner without shock, the entrance edge of the vane must be tangential to the direction of $V_{1}$, and if $\beta_{1}$ be the vane angle at the entrance at the radius $r_{1}$, we obtain

$$
\sin \beta_{1}=\frac{c_{1}}{V_{1}} \sin \alpha_{1}
$$

The flow in the runner is quite artificial, and it may have any form in accordance with that of the vane, it being given arbitrarily. In order, however, to get the best working ability of the runner, the vane should be so formed that the absolute exit velocity $c_{2}$ from it lies in an axial 
plane, or so that the angle $\alpha_{2}$ is equal to $90^{\circ}$ with reference to Fig. 2. Assuming such a condition, the vane angle $\beta_{2}$ at the exit will be given by $\tan \beta_{2}=\frac{c_{2}}{u_{2}}$, or, the assumed turbine being one of an axial flow type, it will be $u_{2}=u_{1}=\omega r_{1}$, and $c_{2}=w_{1}$, and we have

$$
\tan \beta_{2}=\frac{w_{1}}{u_{1}}=\frac{w_{1}}{\omega r_{1}}
$$

and also we obtain

$$
V_{2}=\sqrt{u_{2}^{2}+c_{2}^{2}}=\sqrt{u_{1}^{2}+w_{1}^{2}}
$$

For other kinds of propeller turbines, similar calculations will apply, as the flow in this type of turbines should be straight and direct.

\section{Fundamental Equation for the RunNer}

If the mass $d m$ of water flows through the runner at the radius $r$ in the time $d t$ with the circumferential velocity $v$, the turning moment $d M$ given to the axis of the runner will generally be given by.

$$
d M=d m \frac{d(v r)^{(2)}}{d t}
$$

If $\gamma$ be the density of the water, the mass acting per second at the radius $r_{1}$ will be

$$
\frac{d m}{d t}=\frac{\gamma}{g} 2 \pi r_{1} d r_{1} w_{1}=\frac{\gamma}{g} 2 \pi r_{1} d r_{1} 2 a z_{1},
$$

so that we get

$$
d M=\frac{\gamma}{g} 4 \pi \alpha z_{1} r_{1} d r_{1} d(v r)
$$

Most propeller turbines being an axial flow type, $r_{1}$ is constant throughout the runner, so that $r=r_{1}$, and we have

$$
d M=\frac{\gamma}{g} 4 \pi a z_{1} r_{1}^{2} d r_{1} d v
$$

The. total turning moment $M$ is now

$$
\begin{aligned}
M & =\frac{\gamma}{g} 4 \pi a z_{1} \int_{R^{\prime}}^{R} \int_{v_{2}}^{v_{1}} d v r_{1}^{2} d r_{1}=\frac{\gamma}{g} 4 \pi a z_{1} \int_{R^{\prime}}^{R}\left(v_{1}-v_{2}\right) r_{1}^{2} d r_{1} \\
& =\frac{\gamma}{g} 4 \pi a z_{1} \int_{R^{\prime}}^{R}\left(\frac{k_{1}}{r_{1}}-v_{2}\right) r_{1}^{2} d r_{1}
\end{aligned}
$$

(2) See, for example, H. Lorenz, Technische Hydromechanik, p. $39^{2}$.

Aug. 1928] 


$$
=\frac{\gamma}{g} 4 \pi a z_{1}\left[\frac{k_{1}}{2}\left(R^{2}-R^{\prime 2}\right)-\int_{R^{\prime}}^{R} v_{2} r_{1}^{2} d r_{1}\right] .
$$

If the circumferential velocity $v_{2}$ at the exit edge of the runner be given in a function of the radius $p_{1}$, the above integral can be calculated.

In order to realize the best working condition, we usually design the runner to be $v_{2}=0$ as above mentioned, and in this case the above equation becomes

$$
M=\frac{\gamma}{g} 2 \pi a k_{1} z_{1}\left(R^{2}-R^{\prime 2}\right),
$$

or since $a k_{1}=\frac{Q v_{0} R_{0}}{2 \pi\left(R^{2}-R^{\prime 2}\right) z_{1} \rho_{1}}$ we have

$$
M=\frac{\gamma}{g \rho_{1}} Q v_{0} R_{0}
$$

The power developed by the runner will be, therefore,

$$
\text { Power }=\frac{\gamma}{g \rho_{1}} Q \omega v_{0} R_{0}
$$

or if $u$ be the peripheral velocity of the outer edge of the runner, since $\omega=\frac{u}{R}$, we have

$$
\text { Power }=\frac{\gamma}{g \rho_{1}} Q u v_{0} \frac{P_{0}}{R} .
$$

On the other hand, if $H$ be the total head to be utilized and $\eta$ be the efficiency of the turbine, we have

$$
\text { Power }=\gamma Q_{\eta} H
$$

Hence equating we get the following equation.

$$
u v_{0} \frac{R_{0}}{R}=g \rho_{1} \eta H
$$

which is the fundamental equation for calculating the turbine.

Considering the outermost stream line in the clearance space which is just in contact with the casing, we have with reference to Fig. 1, $R^{2} z_{1}=R_{0}^{2}\left(B_{0}+e\right)$, which gives

$$
\frac{R_{0}}{R}=\sqrt{\frac{z_{1}}{B_{0}+e}}
$$

and, if we substitute this in the above equation we obtain

$$
u v_{0} \sqrt{\frac{z_{1}}{B_{0}+e}}=g \rho_{1} \eta H \text {. }
$$

[Vol. XXXI, No. 136 
For other kinds of propeller turbines, such as for a diagonal turbine, $r$ is a linear function of $r_{1}$, and similar results will easily be obtained.

\section{Graphical Determination of the Form. OF RUNner Vane}

The absolute inflow angle $\alpha_{1}$ to the runner varies as the radius $\eta_{1}$ as shown in equation (16), and all the calculations relating to the vane may be made graphically as shown as follows.-

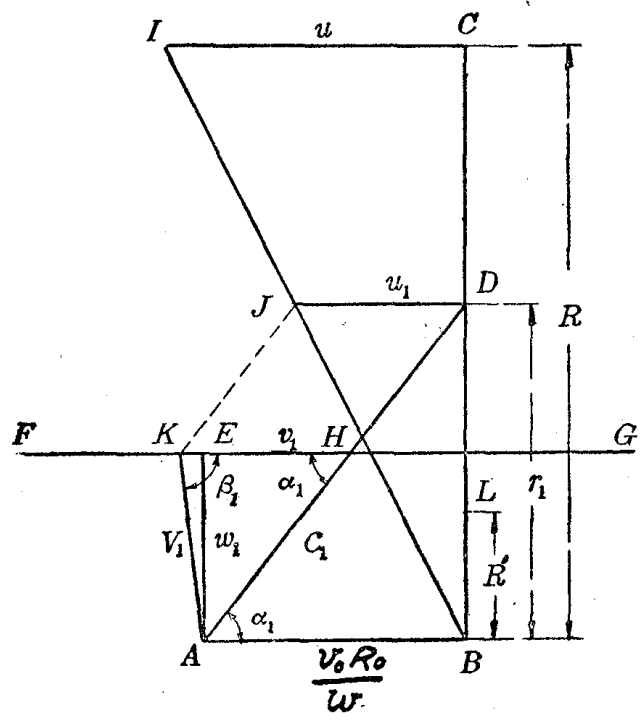

Fig. 3

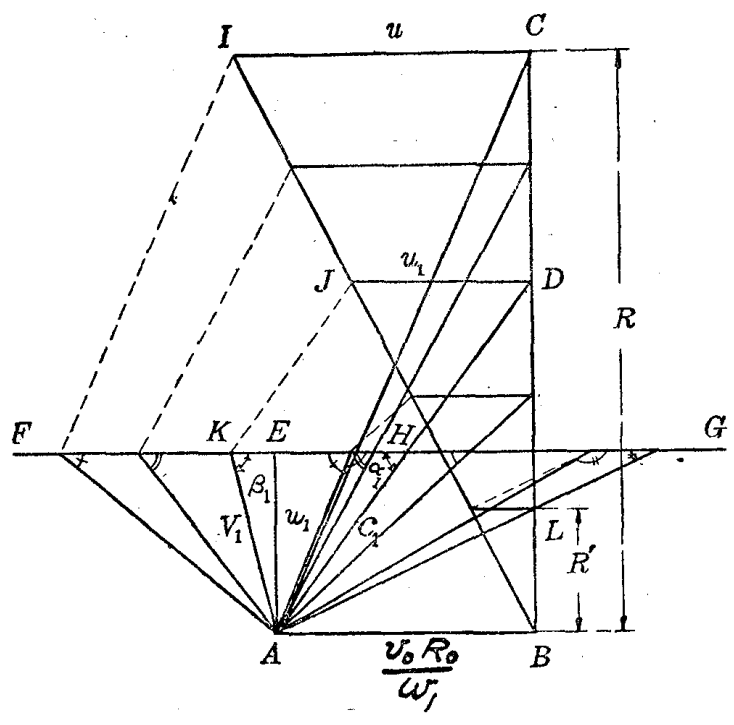

Fig. 4

Referring to Fig. 3, draw $A B$ taking its length equal to the value $\frac{v_{0} R_{0}}{w_{1}}$ with some convenient scale, and draw $B C$ perpendicular to $A B$ taking its length equal to the outer radius $R$ of the runner with the same scale. Take $B D$ equal to the radius $r_{1}$ and join $A$ and $D$, then the angle $B A D$ will give the angle $\alpha_{1}$ at the radius $r_{1}$ as is evident from equation (16).

Draw $A E$ perpendicular to $A B$ taking its length equal to the axial velocity $w_{1}$ at the radius $r_{1}$, and draw $F G$ parallel to $A B$ passing through $\boldsymbol{E}$ and intersecting the line $A D$ at the point $H$. Then we have

$$
H E=A \dot{B} \frac{A E}{B D}=\frac{v_{0} R_{0}}{w_{1}} \cdot \frac{w_{1}}{r_{1}}=\frac{v_{0} R_{0}}{r_{1}}=v_{1}
$$

by equation (13), so that $A H$ will give the absolute inflow velocity $c_{1}$ as is evident from equation (17).

Draw $C I$ perpendicular to $B C$ taking its length equal to the peripheral Aug. 1928] 
velocity $u$ of the outer edge of the runner, and join $B$ and $I$, then the line $D J$ drawn parallel to $C I$ meeting $B I$ at the point $J$ will give the peripheral velocity $u_{1}$ of the runner at the radius $r_{1}$.

Take $H K$ equal to $D J$ and join $A$ and $K$, then $A K$ will represent the relative infow velocity $V_{1}$ and the angle $A K H$ will give the vane angle $\beta_{1}$ at the entrance, both at the radius $r_{1}$ as is obvious from equations (18) and (19).

For an ordinary axial flow propeller runner, in which the axial velocity $w_{1}$ is assumed to be uniform, fixing the length of $A B$ and fixing the line $F G$, repeat the above process varying $B D$ each time between the limits $B L$ and $B C$ as shown in Fig. 4, where $B L$ is the radius $R^{\prime}$ of the hub, then the form of the vane at the entrance edge can easily be determined by this simple graphical method.

At the exit edge of the runner, take $A B$ in Fig. 5 equal to the outer radius $R$ of the runner, and draw $B C$ perpendicular to $A B$ taking its length equal to the corresponding peripheral velocity $u$, and join $A$ and $C$.

Draw $C D$ perdendicular to $B C$ taking its length equal to the axial velocity, and draw $D E$ parallel to $A C$, then a right-angled triangle such as $F G H$ will be the exit velocity triangle at the radius $A F=r_{1}$, and the angle $G F H$ will be the vane a gle $\beta_{2}$ at that radius, the relative outflow velocity $V_{2}$ being represented by $F H$.

The vane angles at the entrance and exit being thus determined at all the corresponding radial points, the next things to consider are how we may join them by smooth curves and how long the curves shall be taken. The consideration has a relation to the number of vanes and the problems are exactly the same as occur in the design of an ordinary reaction turbine.

The above is an example of the graphical

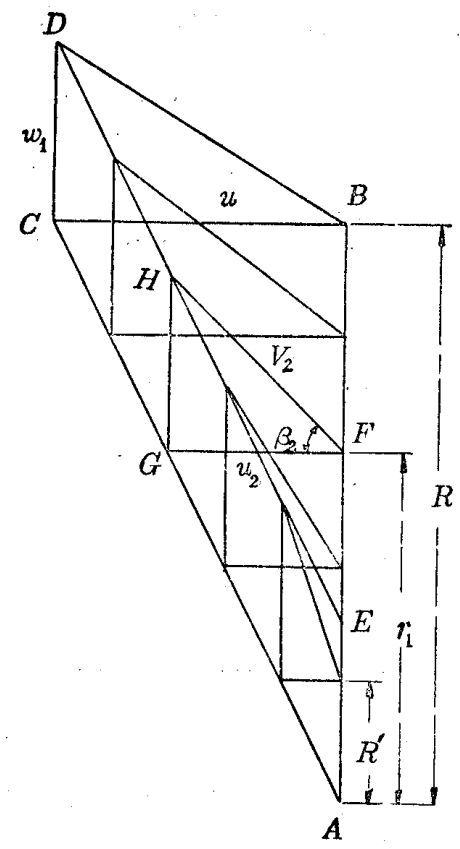

Fig. 5 solution applied to an ordinary axial flow propeller runner, and it is evident that the solution can at once be applied to a diagonal and all the other types of propeller runners.

Sendai, March 23, 1927.

[Vol. XXXI, No. 136 